\title{
Characterization of Nanoparticles for Drug Delivery Applications
}

\author{
Zhengyu Deng, ${ }^{*}$ Ruiping Zhao, ${ }^{* *}$ Liang-Chang Dong, ${ }^{*}$ and Geoff Wong* \\ * ALZA Corp., 1010 Joaquin Road, Mountain View, CA 94043
}

New molecular entities identified by high-throughput screening methods are often poorly watersoluble and thus not easily absorbed in the gastrointestinal tract, due to limitations of dissolution and solubility. There are a variety of approaches to enhancing the drug dissolution and solubility. Particle size reduction is one of these approaches that have been demonstrated as an effective option. Reducing particle size and distribution range increases surface area and speeds up dissolution, thereby facilitating drug absorption. New technologies for obtaining particles in the nanoscale range are being developed [1]. Even so, the ability to control the particles in nanoscale has only recently become part of drug research and development, and much excitement has centered on these technologies' applications for drug delivery [2-4].

Megestrol acetate (MGA), a Class II drug that has poor solubility and high permeability, was chosen as a model compound. Conventional oral dosage forms may not be suitable for the delivery of the drug. Nanosuspension in a self-emulsifying formulation (SEF) will increase the drug payload as well as the bioavailability. However, the stability of nanosuspensions in SEF is a big concern due to Oswald ripening. One approach for improving nanosuspension stability is to coat the nanoparticles with a polymer that is insoluble in the SEF but soluble in water. It is observed that coating MGA nanoparticles with polymer improves the stability of the nanosuspension and prevents the nanoparticles from recrystallization and coagulation/aggregation. It is hypothesized that the nanoparticle morphology and coating efficacy have important roles in the stability of nanosuspension and other performance tests. The Particle Size Analyzer (PSA) has been used to study the particle size distribution at different time intervals. In this study, environmental scanning electron microscope (SEM), high-resolution field emission FE-SEM, and atomic force microscope (AFM) images were collected to provide the morphology information of the nanoparticles. Since AFM provides the ability to investigate the surface structure at nanometer resolution in ambient and liquid environments, the topography of nanoparticles can be further investigated using AFM by immobility of nanoparticles on surfaces [5]. Fourier Transform Infrared Spectroscopy (FTIR) and Raman spectroscopy methods have been developed to investigate the coating efficacy of the different nanoparticle formulations.

In this presentation, we show that the polymers (polyvinylpyrrolidone [PVP], hydroxypropyl methylcellulose [HPMC]) appeared to form a non-covalent absorption layer with MGA. The change of particle morphology provides direct evidence that polymers have been coated on the particle surface. Atomic force microscope topography images also provide additional support for the findings from the SEM study. Clearly, the use of combinations of SEM, FE-SEM, AFM, FTIR, and Raman allow us to investigate the polymer coating of nanoparticles more thoroughly.

\section{References}

[1] J. W. Tom, P. G. Debenedetti, J. Aersol. Sci. 1991, 22, 555-584

[2] R. H. Muller, C. Jacobs, O. Kayser, Adv. Drug Delivery Rev. 2001, 47, 3-19

[3] C. A. Lipinski, Curr. Drug Dis. 2001, 17-19

[4] A. M. Serajuddin, J. Pham. Sci. 1999, 88, 1058-1066

[5] W. A. Duck, T. J. Senden, and R. M. Pashley, Nature 1991, 353, 239 
**Present address: Cytokinetics, Inc., 280 East Grand Avenue, South San Francisco, CA 94080
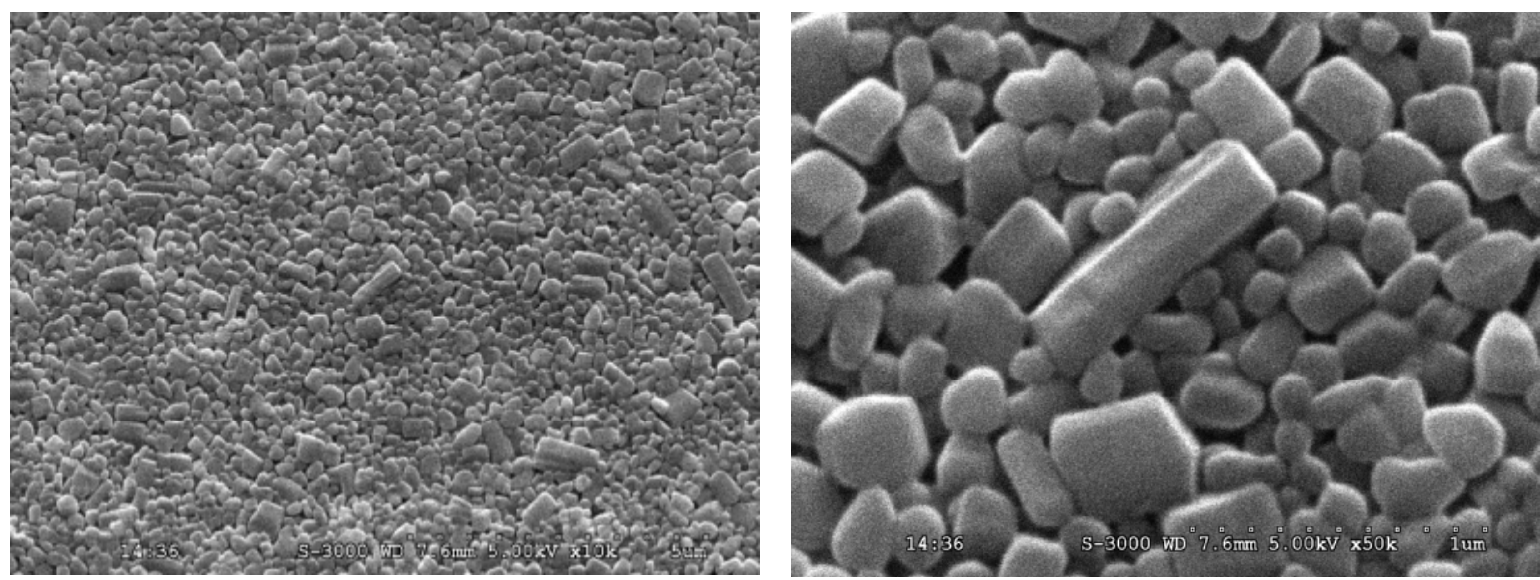

Figure 1. Scanning electron microscope images of nanoparticles of megestrol acetate (MGA)/F108
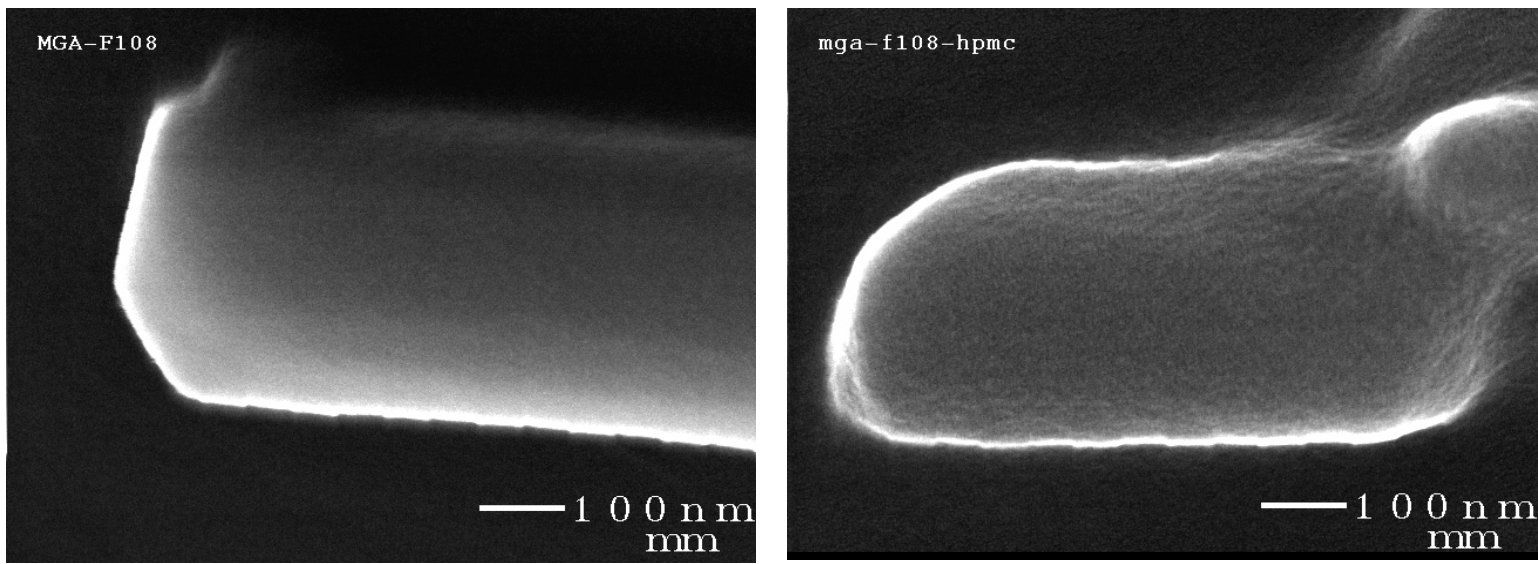

Figure 2. Field emission scanning electronic microscope images of nanoparticle megestrol acetate (MGA)/F108 and MGA/F108/hydroxypropyl methylcellulose (HPMC)
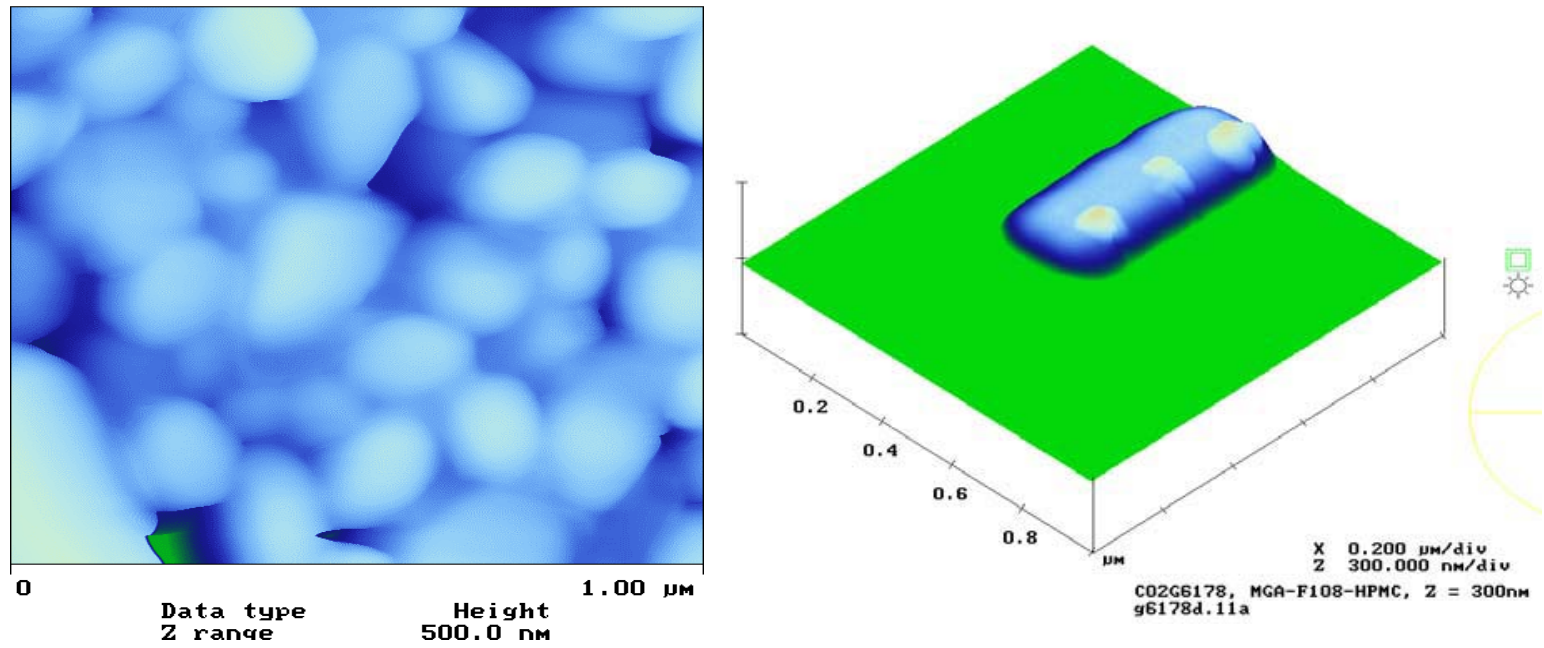

Figure 3. Three-dimensional atomic force microscope image of megestrol acetate (MGA)/F108 and MGA/F108/hydroxypropyl methylcellulose (HPMC) nanoparticles 